\title{
Maximal Green Sequences of Exceptional Finite Mutation Type Quivers ${ }^{\star}$
}

\author{
Ahmet I. SEVEN \\ Middle East Technical University, Department of Mathematics, 06800, Ankara, Turkey \\ E-mail: aseven@metu.edu.tr
}

Received June 18, 2014, in final form August 15, 2014; Published online August 19, 2014

http://dx.doi.org/10.3842/SIGMA.2014.089

\begin{abstract}
Maximal green sequences are particular sequences of mutations of quivers which were introduced by Keller in the context of quantum dilogarithm identities and independently by Cecotti-Córdova-Vafa in the context of supersymmetric gauge theory. The existence of maximal green sequences for exceptional finite mutation type quivers has been shown by Alim-Cecotti-Córdova-Espahbodi-Rastogi-Vafa except for the quiver $X_{7}$. In this paper we show that the quiver $X_{7}$ does not have any maximal green sequences. We also generalize the idea of the proof to give sufficient conditions for the non-existence of maximal green sequences for an arbitrary quiver.
\end{abstract}

Key words: skew-symmetrizable matrices; maximal green sequences; mutation classes

2010 Mathematics Subject Classification: 15B36; 05C50

\section{Introduction and main results}

Maximal green sequences are particular sequences of mutations of quivers. They were used in [9] to study the refined Donaldson-Thomas invariants and quantum dilogarithm identities. Moreover, the same sequences appeared in theoretical physics where they yield the complete spectrum of a BPS particle, see [5, Section 4.2]. The existence of maximal green sequences for exceptional finite mutation type quivers has been shown in [1] except for the quiver $X_{7}$. In this paper, we show that the quiver $X_{7}$ does not have any maximal green sequences. We also give some general sufficient conditions for the non-existence of maximal green sequences for an arbitrary quiver.

To be more specific, we need some terminology. Formally, a quiver is a pair $Q=\left(Q_{0}, Q_{1}\right)$ where $Q_{0}$ is a finite set of vertices and $Q_{1}$ is a set of arrows between them. It is represented as a directed graph with the set of vertices $Q_{0}$ and a directed edge for each arrow. We consider quivers with no loops or 2-cycles and represent a quiver $Q$ with vertices $1, \ldots, n$, by the uniquely associated skew-symmetric matrix $B=B^{Q}$ defined as follows: the entry $B_{i, j}>0$ if and only if there are $B_{i, j}$ many arrows from $j$ to $i$; if $i$ and $j$ are not connected to each other by an edge then $B_{i, j}=0$. We will also consider more general skew-symmetrizable matrices: recall that an $n \times n$ integer matrix $B$ is skew-symmetrizable if there is a diagonal matrix $D$ with positive diagonal entries such that $D B$ is skew-symmetric. To define the notion of a green sequence, we consider pairs $(\mathbf{c}, B)$, where $B$ is a skew-symmetrizable integer matrix and $\mathbf{c}=\left(\mathbf{c}_{1}, \ldots, \mathbf{c}_{n}\right)$ such that each $\mathbf{c}_{i}=\left(c_{1}, \ldots, c_{n}\right) \in \mathbb{Z}^{n}$ is non-zero. Motivated by the structural theory of cluster algebras, we call such a pair $(\mathbf{c}, B)$ a $Y$-seed. Then, for $k=1, \ldots, n$ and any $Y$-seed $(\mathbf{c}, B)$ such that all entries of $\mathbf{c}_{k}$ are non-negative or all are non-positive, the $Y$-seed mutation $\mu_{k}$ transforms $(\mathbf{c}, B)$ into the $Y$-seed $\mu_{k}(\mathbf{c}, B)=\left(\mathbf{c}^{\prime}, B^{\prime}\right)$ defined as follows [8, equation (5.9)], where we use the notation $[b]_{+}=\max (b, 0)$ :

\footnotetext{
${ }^{\star}$ This paper is a contribution to the Special Issue on New Directions in Lie Theory. The full collection is available at http://www.emis.de/journals/SIGMA/LieTheory2014.html
} 
- the entries of the exchange matrix $B^{\prime}=\left(B_{i j}^{\prime}\right)$ are given by

$$
B_{i j}^{\prime}= \begin{cases}-B_{i j} & \text { if } i=k \text { or } j=k, \\ B_{i j}+\left[B_{i k}\right]_{+}\left[B_{k j}\right]_{+}-\left[-B_{i k}\right]_{+}\left[-B_{k j}\right]_{+} & \text {otherwise; }\end{cases}
$$

- the tuple $\mathbf{c}^{\prime}=\left(\mathbf{c}_{1}^{\prime}, \ldots, \mathbf{c}_{n}^{\prime}\right)$ is given by

$$
\mathbf{c}_{i}^{\prime}= \begin{cases}-\mathbf{c}_{i} & \text { if } i=k \\ \mathbf{c}_{i}+\left[\operatorname{sgn}\left(\mathbf{c}_{k}\right) B_{k, i}\right]_{+} \mathbf{c}_{k} & \text { if } i \neq k\end{cases}
$$

The matrix $B^{\prime}$ is skew-symmetrizable with the same choice of $D$. We also use the notation $B^{\prime}=\mu_{k}(B)$ (in (1)) and call the transformation $B \mapsto B^{\prime}$ the matrix mutation. This operation is involutive, so it defines a mutation-equivalence relation on skew-symmetrizable matrices.

We use the $Y$-seeds in association with the vertices of a regular tree. To be more precise, let $\mathbb{T}_{n}$ be an $n$-regular tree whose edges are labeled by the numbers $1, \ldots, n$, so that the $n$ edges emanating from each vertex receive different labels. We write $t \frac{k}{-} t^{\prime}$ to indicate that vertices $t, t^{\prime} \in \mathbb{T}_{n}$ are joined by an edge labeled by $k$. Let us fix an initial seed at a vertex $t_{0}$ in $\mathbb{T}_{n}$ and assign the (initial) $Y$-seed $\left(\mathbf{c}_{0}, B_{0}\right)$, where $\mathbf{c}_{0}$ is the tuple of standard basis. This defines a $Y$-seed pattern on $\mathbb{T}_{n}$, i.e. an assignment of a $Y$-seed $\left(\mathbf{c}_{t}, B_{t}\right)$ to every vertex $t \in \mathbb{T}_{n}$, such that the seeds assigned to the endpoints of any edge $t-t^{\prime}$ are obtained from each other by the seed mutation $\mu_{k}$; we call $\left(\mathbf{c}_{t}, B_{t}\right)$ a $Y$-seed with respect to the initial $Y$-seed $\left(\mathbf{c}_{0}, B_{0}\right)$. We write:

$$
\mathbf{c}_{t}=\mathbf{c}=\left(\mathbf{c}_{1}, \ldots, \mathbf{c}_{n}\right), \quad B_{t}=B=\left(B_{i j}\right) .
$$

We refer to $B$ as the exchange matrix and $\mathbf{c}$ as the $\mathbf{c}$-vector tuple of the $Y$-seed. These vectors have the following sign coherence property [7]:

each vector $\mathbf{c}_{j}$ has either all entries nonnegative or all entries nonpositive.

Note that this property is conjectural if $B$ is a general non-skew-symmetric (but skew-symmetrizable) matrix. It implies, in particular, that the $Y$-seed mutation in (2) is defined for any $Y$-seed $\left(\mathbf{c}_{t}, B_{t}\right)$, furthermore $\mathbf{c}_{t}$ is a basis of $\mathbb{Z}^{n}$ [10, Proposition 1.3]. We also write $\mathbf{c}_{j}>0$ (resp. $\left.\mathbf{c}_{j}<0\right)$ if all entries are non-negative (resp. non-positive).

Now we can recall the notion of a green sequence [3]:

Definition 1. Let $B_{0}$ be a skew-symmetrizable $n \times n$ matrix. A green sequence for $B_{0}$ is a sequence $\mathbf{i}=\left(i_{1}, \ldots, i_{l}\right)$ such that, for any $1 \leq k \leq l$ with $(\mathbf{c}, B)=\mu_{i_{k-1}} \circ \cdots \circ \mu_{i_{1}}\left(\mathbf{c}_{0}, B_{0}\right)$, we have $\mathbf{c}_{i_{k}}>0$, i.e. each coordinate of $\mathbf{c}_{i_{k}}$ is greater than or equal to 0 ; here if $k=1$, then we take $(\mathbf{c}, B)=\left(\mathbf{c}_{0}, B_{0}\right)$. A green sequence for a quiver is a green sequence for the associated skew-symmetric matrix.

A green sequence $\mathbf{i}=\left(i_{1}, \ldots, i_{l}\right)$ is maximal if, for $(\mathbf{c}, B)=\mu_{i_{l}} \circ \cdots \circ \mu_{i_{1}}\left(\mathbf{c}_{0}, B_{0}\right)$, we have $\mathbf{c}_{k}<0$ for all $k=1, \ldots, n$.

In this paper, we study the maximal green sequences for the quivers which are mutationequivalent to the quiver $X_{7}$ (Fig. 1). Our result is the following:

Theorem 1. Suppose that $Q$ is mutation-equivalent to the quiver $X_{7}$ (so $Q$ is one of the quivers in Fig. 1). Then $Q$ does not have any maximal green sequences.

We prove the theorem using the following general statement, which can be easily checked to give a sufficient condition for the non-existence of maximal green sequences: 

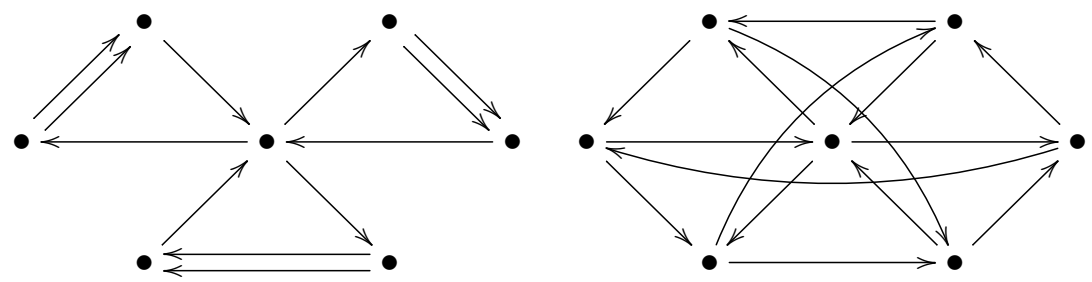

Figure 1. Quivers which are mutation-equivalent to $X_{7}$; the first one is the quiver $X_{7}$, see [6].

Proposition 1. Let $B_{0}$ be a skew-symmetrizable initial exchange matrix. Suppose that there is a vector $u>0$ such that, for any $Y$-seed $(\mathbf{c}, B)$ with respect to the initial seed $\left(\mathbf{c}_{0}, B_{0}\right)$, the coordinates of $u$ with respect to $\mathbf{c}$ are non-negative. Then, under assumption (3), the matrix $B_{0}$ does not have any maximal green sequences.

We establish such a vector for the quiver $X_{7}$ :

Proposition 2. Suppose that $Q_{0}$ is a quiver which is mutation-equivalent to $X_{7}$, so $Q_{0}$ is one of the quivers in Fig. 1 , and $B_{0}$ is the corresponding skew-symmetric matrix. Let $u=$ $\left(a_{1}, a_{2}, \ldots, a_{7}\right)$ be the vector defined as follows:

(*) if $Q_{0}$ is the quiver $X_{7}$ (so $Q$ is the first quiver in Fig. 1), then the coordinate corresponding to the "center" is equal 2 , and the rest is equal to 1 ; if $Q_{0}$ is not the quiver $X_{7}$ (so $Q$ is the second quiver in Fig. 1), then all coordinates are equal to 1.

Then, for any $Y$-seed $(\mathbf{c}, B)$ with respect to the initial seed $\left(\mathbf{c}_{0}, B_{0}\right)$, the coordinates of $u$ with respect to $\mathbf{c}$ is of the same form as in $(*)$. In particular, the coordinates of $u$ with respect to $\mathbf{c}$ are positive.

(The vector $u$ is a radical vector for $B_{0}$, i.e. $B_{0} u=0$. In fact, any radical vector for $B_{0}$ is a multiple of $u$.)

We generalize this statement to an arbitrary quiver as follows:

Theorem 2. Let $B_{0}$ be a skew-symmetrizable initial exchange matrix and suppose that $u_{0}>0$ is a radical vector for $B_{0}$, i.e. $B_{0} u_{0}=0$. Suppose also that, for any $Y$-seed $(\mathbf{c}, B)$ with respect to the initial seed $\left(\mathbf{c}_{0}, B_{0}\right)$, the coordinates of $u_{0}$ with respect to $\mathbf{c}$ are non-negative. Then, under assumption (3), for any $B$ which is mutation-equivalent to $B_{0}$, the matrix $B$ does not have any maximal green sequences.

We prove our results in Section 2. For related applications of maximal green sequences, we refer the reader to [4] and [11].

\section{Proofs of main results}

Let us first note how the coordinates of a vector change under the mutation operation, which can be easily checked using the definitions (assuming (3)):

Proposition 3. Suppose that $(\mathbf{c}, B)$ is a $Y$-seed with respect to an initial $Y$-seed. Suppose also that the coordinate vector of $u$ with respect to $\mathbf{c}$ is $\left(a_{1}, \ldots, a_{n}\right)$. Let $\left(\mathbf{c}^{\prime}, B^{\prime}\right)=\mu_{k}(\mathbf{c}, B)$ and $\left(a_{1}^{\prime}, \ldots, a_{n}^{\prime}\right)$ be the coordinates of $u$ with respect to $\mathbf{c}^{\prime}$. Then $a_{i}=a_{i}^{\prime}$ if $i \neq k$ and $a_{k}^{\prime}=$ $-a_{k}+\sum a_{i}\left[\operatorname{sgn}\left(\mathbf{c}_{k}\right) B_{k, i}\right]_{+}$, where the sum is over all $i \neq k$. 
As we mentioned, in view of Proposition 1, Theorem 1 follows from Proposition 2. To prove Proposition 2, it is enough to show that the coordinates of the vector $u$ change as stated, i.e. show that if the coordinates of $u$ with respect to $\mathbf{c}$ are as in $(*)$, then for the $Y$-seed $\left(\mathbf{c}^{\prime}, B^{\prime}\right)=\mu_{k}(\mathbf{c}, B)$, the coordinates with respect to $\mathbf{c}^{\prime}$ are also of the form in $(*)$. This can be checked easily using the formula in Proposition 3.

To prove Theorem 2, let us first note the following property of the coordinates of the radical vectors:

Lemma 1. Suppose that $(\mathbf{c}, B)$ is a $Y$-seed with respect to an initial $Y$-seed $\left(\mathbf{c}_{0}, B_{0}\right)$ and $u_{0}$ is a radical vector for $B_{0}$. Suppose that the coordinate vector of $u_{0}$ with respect to $\mathbf{c}$ is $\left(a_{1}, \ldots, a_{n}\right)$. Then, for any index $k$, we have the following:

$$
\sum a_{i}\left[\operatorname{sgn}\left(\mathbf{c}_{k}\right) B_{k, i}\right]_{+}=\sum a_{i}\left[-\operatorname{sgn}\left(\mathbf{c}_{k}\right) B_{k, i}\right]_{+},
$$

where the sum is over all $i \neq k$.

In particular, for radical vectors, the formula in Proposition 3 that describe the change of coordinates under mutation depends only on the exchange matrix, not on the c-vectors.

To prove the lemma, suppose that $D=\operatorname{diag}\left(d_{1}, \ldots, d_{n}\right)$ is a skew-symmetrizing matrix for $B_{0}$, so it is also skew-symmetrizing for $B$, so $D B=C$ is skew-symmetric, i.e. $C_{i, k}=d_{i} B_{i, k}=$ $-d_{k} B_{k, i}=-C_{k, i}$ for all $i, k$. Let $u=\left(a_{1}, \ldots, a_{n}\right)$. Then $u$ is a radical vector for $B$, so it is also a radical vector for $C=D B$, i.e. $C u=0$, which means that for any index $k$, we have $\sum a_{i}\left[\operatorname{sgn}\left(\mathbf{c}_{k}\right) C_{k, i}\right]_{+}=\sum a_{i}\left[\operatorname{sgn}\left(\mathbf{c}_{k}\right) C_{i, k}\right]_{+}$, which is equal to $\sum a_{i}\left[-\operatorname{sgn}\left(\mathbf{c}_{k}\right) C_{k, i}\right]_{+}$, where the sum is over all $i \neq k$. Then, writing $C_{k, i}=d_{k} B_{k, i}$, we have

$$
\sum a_{i}\left[\operatorname{sgn}\left(\mathbf{c}_{k}\right) d_{k} B_{k, i}\right]_{+}=\sum a_{i}\left[-\operatorname{sgn}\left(\mathbf{c}_{k}\right) d_{k} B_{k, i}\right]_{+} .
$$

Dividing both sides by $d_{k}>0$, we obtain the lemma.

We will also need the following property of the radical vectors:

Lemma 2. In the set-up of Theorem 2 , let $u$ denote the vector which represents $u_{0}$ with respect to the basis $\mathbf{c}$. Then $u$ is a radical vector for $B$, i.e. $B u=0$.

To prove the lemma, let us note that $u$ can be obtained from $u_{0}$ by applying the formula in Proposition 3 along with the mutations. Thus, to prove the lemma, it is enough to show that, for any $k=1, \ldots, n$, we have the following:

$(* *)$ if $u=\left(a_{1}, \ldots, a_{n}\right)$ is a radical vector for $B$, then $u^{\prime}$ is a radical vector for $B^{\prime}=\mu_{k}(B)$, i.e. $B^{\prime} u^{\prime}=0$, where $u^{\prime}=\left(a_{1}^{\prime}, \ldots, a_{n}^{\prime}\right)$ is the vector as in Proposition 3.

To show $(* *)$, we write $B^{\prime}$ in matrix notation as follows [2, Lemma 3.2]: for $\epsilon=\operatorname{sgn}\left(\mathbf{c}_{k}\right)$, we have

$$
B^{\prime}=\left(J_{n, k}+E_{k}\right) B\left(J_{n, k}+F_{k}\right),
$$

where

- $J_{n, k}$ denotes the diagonal $n \times n$ matrix whose diagonal entries are all 1's, except for -1 in the $k$ th position;

- $E_{k}$ is the $n \times n$ matrix whose only nonzero entries are $e_{i k}=\left[-\varepsilon b_{i k}\right]_{+}$;

- $F_{k}$ is the $n \times n$ matrix whose only nonzero entries are $f_{k j}=\left[\varepsilon b_{k j}\right]_{+}$. 
It follows from a direct check that $\left(J_{n, k}+F_{k}\right) u^{\prime}=u$. Then $B^{\prime} u^{\prime}=\left(J_{n, k}+E_{k}\right) B\left(J_{n, k}+F_{k}\right) u^{\prime}=$ $\left(J_{n, k}+E_{k}\right) B u=\left(J_{n, k}+E_{k}\right) 0=0$. This completes the proof of the lemma.

Let us now prove Theorem 2. For this, we first consider the $Y$-seed pattern defined by the initial $Y$-seed $\left(\mathbf{c}_{0}, B_{0}\right)$ at the initial vertex $t_{0}$. Let us suppose that $t_{1}$ is a vertex such that the corresponding $Y$ seed $(\mathbf{c}, B)$ has the exchange matrix $B$. Then we can consider the $Y$-seed pattern defined by the initial $Y$-seed $\left(\mathbf{c}_{0}, B\right)$ at the initial vertex $t_{1}$ (where $\mathbf{c}_{0}$ is the tuple of standard basis). Then we have the following: for any fixed vertex $t$ of the $n$-regular tree $\mathbb{T}_{n}$, the exchange matrices of the $Y$-seeds assigned by these patterns coincide because the pattern is formed by mutating at the labels of the $n$-regular tree $\mathbb{T}_{n}$ and mutation is an involutive operation on matrices; let us denote these seeds by $\left(\mathbf{c}^{\prime}, B^{\prime}\right)$ and $\left(\mathbf{c}^{\prime \prime}, B^{\prime}\right)$ respectively.

On the other hand, let $u$ denote the vector which represents $u_{0}$ with respect to the basis $\mathbf{c}$, which can be obtained by applying the formula in Proposition 3 along with the mutations. Then $u$ is a radical vector for $B$, i.e. $B u=0$ (Lemma 2). Furthermore, the coordinates of the vectors $u_{0}$ and $u$ with respect to the bases $\mathbf{c}^{\prime}$ and $\mathbf{c}^{\prime \prime}$ respectively will coincide by Lemma 1 (which says that for radical vectors the formula in Proposition 3 depends only on the exchange matrices, not on the c-vectors). In particular, the coordinates of $u$ with respect to any basis of $\mathbf{c}$-vectors are non-negative. Thus, by Proposition 1 , the matrix $B$ does not have any maximal green sequences. This completes the proof.

\section{Acknowledgements}

The author's research was supported in part by the Scientific and Technological Research Council of Turkey (TUBITAK) grant \# 113F138. The author also thanks Christoff Geiss for drawing his attention to the paper [1] by presenting its results at the Workshop on Hall and Cluster Algebras in CRM, University of Montreal. He also thanks the organizers for organizing the conference.

\section{References}

[1] Alim M., Cecotti S., Córdova C., Espahbodi S., Rastogi A., Vafa C., BPS quivers and spectra of complete $\mathcal{N}=2$ quantum field theories, Comm. Math. Phys. 323 (2013), 1185-1227, arXiv:1109.4941.

[2] Berenstein A., Fomin S., Zelevinsky A., Cluster algebras. III. Upper bounds and double Bruhat cells, Duke Math. J. 126 (2005), 1-52, math.RT/0305434.

[3] Brüstle T., Dupont G., Pérotin M., On maximal green sequences, Int. Math. Res. Not. 2014 (2014), 45474586, arXiv:1205.2050.

[4] Canakci I., Lee K., Schiffler R., On cluster algebras from unpunctured surfaces with one marked point, arXiv:1407.5060.

[5] Cecotti S., Córdova C., Vafa C., Braids, walls and mirrors, arXiv:1110.2115.

[6] Derksen H., Owen T., New graphs of finite mutation type, Electron. J. Combin. 15 (2008), 139, 15 pages, arXiv:0804.0787.

[7] Derksen H., Weyman J., Zelevinsky A., Quivers with potentials and their representations II: Applications to cluster algebras, J. Amer. Math. Soc. 23 (2010), 749-790, arXiv:0904.0676.

[8] Fomin S., Zelevinsky A., Cluster algebras. IV. Coefficients, Compos. Math. 143 (2007), 112-164, math.RA/0602259.

[9] Keller B., On cluster theory and quantum dilogarithm identities, in Representations of Algebras and Related Topics, EMS Ser. Congr. Rep., Eur. Math. Soc., Zürich, 2011, 85-116, arXiv:1102.4148.

[10] Nakanishi T., Zelevinsky A., On tropical dualities in cluster algebras, in Algebraic Groups and Quantum Groups, Contemp. Math., Vol. 565, Amer. Math. Soc., Providence, RI, 2012, 217-226, arXiv:1101.3736.

[11] Seven A.I., Maximal green sequences of skew-symmetrizable $3 \times 3$ matrices, Linear Algebra Appl. 440 (2014), 125-130, arXiv:1207.6265. 